The Astrophysical Journal, 619:L63-L66, 2005 January 20

(C) 2005. The American Astronomical Society. All rights reserved. Printed in U.S.A.

\title{
GALAXY EVOLUTION EXPLORER ULTRAVIOLET SPECTROSCOPY AND DEEP IMAGING OF LUMINOUS INFRARED GALAXIES IN THE EUROPEAN LARGE-AREA ISO SURVEY S1 FIELD
}

\author{
Denis Burgarella, ${ }^{1}$ Véronique Buat,${ }^{1}$ Todd Small, ${ }^{2}$ Tom A. Barlow, ${ }^{2}$ Samuel Boissier, ${ }^{3}$ Armando Gil de Paz, ${ }^{3}$ \\ Timothy M. Heckman, ${ }^{4}$ Barry F. Madore,${ }^{3,5}$ D. Christopher Martin, ${ }^{2}$ R. Michael Rich,${ }^{6}$ Luciana Bianchi, ${ }^{7}$ \\ Yong-Ik Byun,${ }^{8}$ Jose Donas, ${ }^{1}$ Karl Forster, ${ }^{2}$ Peter G. Friedman, ${ }^{2}$ Patrick N. Jelinsky, ${ }^{6,9}$ \\ Young-Wook Lee, ${ }^{5,8}$ Roger F. Malina, ${ }^{1}$ Bruno Milliard, ${ }^{1}$ Patrick Morrissey, ${ }^{2}$ \\ Susan G. NefF, ${ }^{7,10}$ David Schiminovich, ${ }^{2}$ Oswald H. W. Siegmund, ${ }^{6,9}$ \\ Alex S. Szalay, ${ }^{4}$ Barry Y. Welsh,${ }^{6,9}$ AND Ted K. Wyder ${ }^{2}$ \\ Received 2004 June 7; accepted 2004 July 2; published 2005 January 17
}

\begin{abstract}
The European Large-Area ISO Survey (ELAIS) S1 field was observed by the Galaxy Evolution Explorer $(G A L E X)$ in both its Wide Spectroscopic and Deep Imaging Survey modes. This field was previously observed by the Infrared Space Observatory, and we made use of the catalog of multiwavelength data published by the ELAIS consortium to select galaxies common to the two samples. Among the 959 objects with GALEX spectroscopy, 88 are present in the ELAIS catalog and 19 are galaxies with an optical spectroscopic redshift. The distribution of redshifts covers the range $0<z<1.6$. The selected galaxies have bolometric IR luminosities $10<\log L_{\mathrm{IR}}<13$ (deduced from the $15 \mu \mathrm{m}$ flux using ISOCAM), which means that we cover a wide range of galaxies from normal to ultraluminous IR galaxies. The mean $(\sigma)$ UV luminosity (not corrected for extinction) amounts to $\log \lambda L_{1530}=9.8(0.6) L_{\odot}$ for the low- $z(z \leq 0.35)$ sample. The UV slope $\beta$ (assuming $f_{\lambda} \propto \lambda^{\beta}$ ) correlates with the GALEX FUV - NUV color if the sample is restricted to galaxies below $z<0.1$. Taking advantage of the UV and IR data, we estimate the dust attenuation from the IR/UV ratio and compare it to the UV slope $\beta$. We find that it is not possible to uniquely estimate the dust attenuation from $\beta$ for our sample of galaxies. These galaxies are highly extinguished with a median value $A_{\mathrm{Fuv}}=2.7 \pm 0.8$. Once the dust correction is applied, the UV- and IR-based star formation rates correlate. For the closest galaxy with the best quality spectrum, we see a feature consistent with being produced by a bump near $220 \mathrm{~nm}$ in the attenuation curve.
\end{abstract}

Subject headings: dust, extinction — galaxies: starburst — infrared: galaxies — ultraviolet: galaxies

\section{INTRODUCTION}

The Galaxy Evolution Explorer (GALEX) is a $50 \mathrm{~cm}$ telescope devoted to observing the ultraviolet (UV; in two bands centered at about $153 \mathrm{~m}$ and $231 \mathrm{~nm}$ ) sky in several imaging surveys including an All-Sky Imaging Survey and several spectroscopic surveys (Martin et al. 2005). This Letter presents the first analysis of the GALEX early release spectroscopic obser-

\footnotetext{
${ }^{1}$ Observatoire Astronomique Marseille Provence, Laboratoire d'Astrophysique de Marseille, traverse du siphon, 13376 Marseille cedex 12, France; denis.burgarella@oamp.fr, veronique.buat@oamp.fr, jose.donas@oamp.fr, roger .malina@oamp.fr, bruno.milliard@oamp.fr.

${ }^{2}$ California Institute of Technology, MC 405-47, 1200 East California Boulevard, Pasadena, CA 91125; tas@srl.caltech.edu, tab@srl.caltech.edu,cmartin@ srl.caltech.edu, krl@srl.caltech.edu, friedman@srl.caltech.edu, patrick@srl .caltech.edu,ds@srl.caltech.edu,wyder@srl.caltech.edu.

${ }^{3}$ Observatories of the Carnegie Institution of Washington, 813 Santa Barbara Street, Pasadena, CA 91101; boissier@ociw.edu, agpaz@ipac.caltech.edu, barry@ipac.caltech.edu.

${ }^{4}$ Department of Physics and Astronomy, Johns Hopkins University, 3400 North Charles Street, Baltimore, MD 21218; heckman@adcam.pha.jhu.edu, szalay@tardis.pha.jhu.edu.

${ }^{5}$ NASA/IPAC Extragalactic Database, California Institute of Technology, Mail Code 100-22, 770 South Wilson Avenue, Pasadena, CA 91125.

${ }^{6}$ Department of Physics and Astronomy, University of California, Los Angeles, CA 90065; rmr@astro.ucla.edu.

${ }^{7}$ Center for Astrophysical Sciences, Johns Hopkins University, 3400 North Charles Street, Baltimore, MD 21218; bianchi@skyrv.pha.jhu.edu.

${ }^{8}$ Center for Space Astrophysics, Yonsei University, Seoul 120-749, Korea; byun@csa.yonsei.ac.kr,ywlee@csa.yonsei.ac.kr.

${ }^{9}$ Space Sciences Laboratory, University of California at Berkeley, 601 Campbell Hall, Berkeley, CA 94720; patj@ssl.berkeley.edu, ossy@ssl.berkeley.edu,bwelsh@ ssl.berkeley.edu.

${ }^{10}$ Laboratory for Astronomy and Solar Physics, NASA Goddard Space Flight Center, Greenbelt, MD 20771; neff@ cobblr.gsfc.nasa.gov.
}

vations available from GALEX. The European Large-Area ISO Survey (ELAIS) is a database of deep $15 \mu \mathrm{m}$ observations carried out with the Infrared Space Observatory (ISO) to study the extragalactic universe in the IR range. The correlation of ELAIS with UV data allows us to carry out a multiwavelength study and to estimate the dust attenuation to deduce and compare star formation rates (SFRs) estimated from the UV and IR. Applications of this work are related to Lyman break galaxies discovered at $z=3$ (Steidel et al. 1996; Lowenthal et al. 1997) and now to even higher redshift galaxies at $z \approx 6$ with the Hubble Ultra Deep Field by Bunker et al. (2004) and maybe $z=10$ (Pello et al. 2004). Although the present sample is not purely UV-selected, it aims at better understanding how to correct for the dust attenuation in these galaxies. It could also lead to a way of understanding UV characteristics of IRbright galaxies.

We assume a cosmology with $H_{0}=70 \mathrm{~km} \mathrm{~s}^{-1} \mathrm{Mpc}^{-1}$, $\Omega_{M}=0.3$, and $\Omega_{\mathrm{VAC}}=0.7$ in this Letter.

\section{THE GALAXY SAMPLE}

GALEX observed the ELAIS S1 field for $12,198 \mathrm{~s}$ in the imaging mode on 2003 September 15 and 31,267 s in the spectroscopic mode on 2003 September 24. This field had already been observed by ISO using ISOCAM. The southern field, S1, centered at $\alpha=00^{\mathrm{h}} 34^{\mathrm{m}} 44.4, \quad \delta=-43^{\circ} 28^{\prime} 12^{\prime \prime} \quad(\mathrm{J} 2000.0)$, covers an area of the sky of about $2 \times 2 \mathrm{deg}^{2}$. The final bandmerged ELAIS catalog (Rowan-Robinson et al. 2004), containing 3523 sources, is available through the ELAIS Web site. The S1 catalog (complete at the $5 \sigma$ level) contains 462 sources in the flux density range $0.5-150 \mathrm{mJy}$ (La Franca et al. 2004). The 
TABLE 1

Multiwavelength Analysis of Selected Galaxies

\begin{tabular}{|c|c|c|c|c|c|c|c|c|c|}
\hline Galaxy Name & $\mathrm{FUV}^{\mathrm{a}}$ & $\mathrm{NUV}^{\mathrm{b}}$ & $\beta\left(\sigma_{\beta}\right)$ & $\begin{array}{l}\log L_{\mathrm{FUV}} \\
\left(\operatorname{ergs~s}^{-1}\right)\end{array}$ & $\begin{array}{c}\log L_{\mathrm{IR}}{ }^{\mathrm{c}} \\
\left(\operatorname{ergs~s}^{-1}\right)\end{array}$ & $z_{\text {spec }}{ }^{\mathrm{d}}$ & $A_{\mathrm{FUV}}$ & $\mathrm{SFR}_{\mathrm{UV}}$ & $\mathrm{SFR}_{\mathrm{IR}}$ \\
\hline ELAISC15 J003828-433848 & 17.7 & 17.4 & $-1.58(0.04)$ & 9.9 & 11.1 & 0.048 & 2.43 & 19.3 & 19.6 \\
\hline ELAISC15 J003731-440812 & 18.7 & 18.4 & $-1.25(0.05)$ & 9.6 & 10.6 & $0.052^{\mathrm{e}}$ & 2.06 & $\ldots$ & $\ldots$ \\
\hline ELAISC15 J003645-440720 ..... & 18.4 & 18.1 & $-1.50(0.08)$ & 9.8 & 10.3 & 0.059 & 1.17 & 5.5 & 3.8 \\
\hline ELAISC15 J003926-441140 & 20.0 & 19.5 & $-0.98(0.06)$ & 9.5 & 11.2 & $0.088^{\mathrm{e}}$ & 3.22 & ... & ... \\
\hline ELAISC15 J003859-433936 ..... & 20.0 & 19.7 & $-1.42(0.12)$ & 9.8 & 10.6 & 0.119 & 1.67 & 8.4 & 9.2 \\
\hline ELAISC15 J003938-433755 ..... & 21.9 & 21.3 & $-1.54(0.23)$ & 9.1 & 10.9 & $0.125^{\mathrm{e}}$ & 3.61 & $\ldots$ & $\ldots$ \\
\hline ELAISC15 J003530-435604 ...... & 22.0 & 21.2 & $-1.66(0.28)$ & 9.2 & 11.2 & 0.147 & 3.90 & 16.9 & 25.3 \\
\hline ELAISC15 J003603-435602 …. & 21.4 & 20.8 & $-1.42(0.24)$ & 9.5 & 11.0 & 0.148 & 2.99 & 12.5 & 4.5 \\
\hline ELAISC15 J003942-435403 & 21.7 & 21.2 & $-1.35(0.12)$ & 9.4 & 10.7 & 0.149 & 2.61 & 6.8 & 9.4 \\
\hline ELAISC15 J003932-441130 ..... & 20.2 & 19.5 & $-1.23(0.11)$ & 10.2 & 11.5 & 0.185 & 2.59 & 43.9 & 43.9 \\
\hline ELAISC15 J003921-441134. & 22.0 & 21.2 & $-1.87(0.23)$ & 9.5 & 11.1 & 0.189 & 3.15 & 15.1 & 19.6 \\
\hline ELAISC15 J003754-441106 ..... & 21.1 & 20.4 & $-1.71(0.23)$ & 10.0 & 11.7 & 0.212 & 3.58 & 46.0 & 38.8 \\
\hline ELAISC15 J003721-434239 & 20.9 & 20.5 & $-1.23(0.14)$ & 10.1 & 11.9 & $0.225^{\mathrm{e}}$ & 3.69 & $\ldots$ & $\ldots$ \\
\hline ELAISC15 J003716-434153 ..... & 21.2 & 20.3 & $-1.22(0.24)$ & 10.0 & 11.0 & 0.226 & 2.04 & 14.8 & 15.9 \\
\hline ELAISC15 J003531-434448 & 20.9 & 20.1 & $-2.04(0.27)$ & 10.3 & 11.0 & 0.286 & 1.46 & 25.5 & 14.9 \\
\hline ELAISC15 J003526-435640 ..... & 21.9 & 21.2 & $\ldots$ & 10.0 & 11.5 & 0.324 & 2.91 & 44.0 & 57.9 \\
\hline ELAISC15 J003954-440510 ..... & 21.4 & 21.1 & $-1.90(0.31)$ & 10.3 & 11.8 & 0.331 & 3.15 & $\ldots$ & $\ldots$ \\
\hline ELAISC15 J003813-433315 ..... & 21.0 & 19.6 & $\ldots$ & 12.0 & 12.9 & $1.400^{\mathrm{f}}$ & $\ldots$ & $\ldots$ & $\ldots$ \\
\hline ELAISC15 J003829-434454 ..... & $\ldots$ & 19.3 & $\ldots$ & $\ldots$ & 13.8 & $1.567^{\mathrm{f}}$ & $\ldots$ & $\ldots$ & 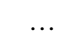 \\
\hline
\end{tabular}

Notes. - UV slopes $\beta$ are estimated from the available rest-frame range (i.e., $\lambda \lambda 1200-3000$ ) except for ELAISC15 J003526-435640, which is at the edge of the field of view, and the two last objects, for which this rest-frame range is not observed by GALEX because

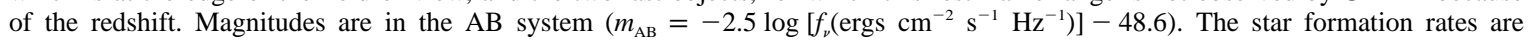
estimated from Kennicutt (1998) and $A_{\mathrm{FUv}}$ from Buat et al. (2002).

${ }^{\text {a }}$ Relative error in FUV amounts to about 0.10 .

${ }^{\mathrm{b}}$ Relative error in NUV amounts to about 0.05 .

c Bolometric IR luminosities are computed following Chary \& Elbaz (2001). Absolute errors should be about 0.5 in $\log L_{\mathrm{IR}}$.

${ }^{\mathrm{d}}$ Spectroscopic redshifts from the ELAIS database.

${ }^{\mathrm{e}}$ Seyfert 2 galaxies from the ELAIS database.

${ }^{\mathrm{f}}$ Seyfert 1 galaxies from the ELAIS database.

GALEX IR0.2 pipeline detected 959 spectra within the 1.2 field of view that we cross-correlated with the ELAIS S1 field. The area of the common field is about $0.5 \mathrm{deg}^{2}$. Our ELAIS S1 sample was selected by cross-correlating the UV GALEX spectroscopic sample with the mid-IR (MIR; at $15 \mu \mathrm{m}) I S O$ catalog assuming that the emission from objects within a circular aperture of 6 " radius could be from the same object. This cross-correlation provided us with 88 objects, most of which proved to be Galactic foreground stars. From this sample, 19 galaxies with spectroscopic redshifts were finally selected (Table 1), and we focus on this sample to carry out a multiwavelength analysis. All the objects except two are below $z=0.35$ : their average far-UV (FUV) luminosity is $\log L_{\mathrm{FUV}}=9.8( \pm 0.6) L_{\odot}$, and their average IR luminosity is $\log L_{\mathrm{IR}}=11.1( \pm 0.5) L_{\odot}$, where $\log L$ are luminosities in units of ergs per second. If we assume luminous IR galaxies (LIRGs) have luminosities in the range $10^{11} \leq L_{\mathrm{IR}} / L_{\odot}<10^{12}$ and ultraluminous IR galaxies (ULIRGs) have $L_{\mathrm{IR}} / L_{\odot}>10^{12}$ (Chary \& Elbaz 2001; Goldader et al. 2002), 12 of them qualify as LIRGs, four objects are Seyfert 1 galaxies, and one object is classified as a LINER. Two objects are at $z>1$; their UV luminosities are above $\log L_{\mathrm{UV}}=12$, and their IR luminosities are about $\log L_{\mathrm{IR}}=13$, i.e., ULIRGs. However, since they are classified by ELAIS as Seyfert 1, the contribution of the central nonthermal QSO is very likely to be predominant for these objects, and their study is outside the scope of the present Letter, which is to study star-forming objects and their dust attenuation.

\section{GALEX UV COLORS AND THE UV SLOPE $\beta$}

The interpretation of UV spectra must mainly deal with two parameters: the stellar population (with associated parameters: SFR, age, metallicity, etc.) and the dust reddening (with associated parameters: attenuation curve, amount of dust, etc.). In order to get back to the former, we have to correct for the latter. To estimate this correction, Calzetti et al. (1994) fitted the observed UV continuum (corrected for the foreground extinction) by a power law expressed as $f_{\lambda} \propto \lambda^{\beta}$ in the range $1250 \leq$ $\lambda(\AA) \leq 2600$. They selected windows to exclude absorption lines and the $2175 \AA$ dust feature, which might cause a departure from the assumed power law. For their sample of starbursts, the resulting $\beta$ was found to correlate with the Balmer optical depth and therefore with the dust content. One interesting point is that the attenuation curve deduced from the Calzetti et al. (1994) sample does not show any evidence from the $2175 \AA$ dust feature.

We estimated the UV slope $\beta$ of our sample after visually inspecting the spectra and discarding pixels in absorption lines or where the signal-to-noise ratio $(\mathrm{S} / \mathrm{N})$ was low. Following Calzetti et al. (1994), we assumed that no $2175 \AA$ dust feature is present and keep the corresponding pixels in the fit. The slopes obtained from the fit over the left pixels are listed in Table 1 with the associated uncertainty $\sigma_{\beta}$. Figure 1 presents one of the best and one of the worst fits over the observed spectra. For ELAISC15 J0035-4356, lying at the edge of the field of view and close to a bright object, the slope cannot be estimated. For most of the GALEX database, we have the UV color FUV - NUV, which can be used (Buat et al. 2005; Schiminovich et al. 2005; Arnouts et al. 2005) to evaluate the UV slope $\beta$. So, it is interesting to compare the estimated $\beta$ to the FUV - NUV colors (Fig. 2). There is no apparent relationship between the GALEX FUV - NUV color and the UV slope $\beta$ for the complete sample. However, as shown by the analysis of Far Ultraviolet Spectroscopic Explorer spectra by Buat et al. (2002) and expected from models (e.g., Leitherer et al. 1999), the continuum of starburst galaxies flattens below $\sim 1200 \AA$ and it cannot be fitted over the full 912-2400 $\AA$ by a single power law. If we restrict our sample to galaxies with 


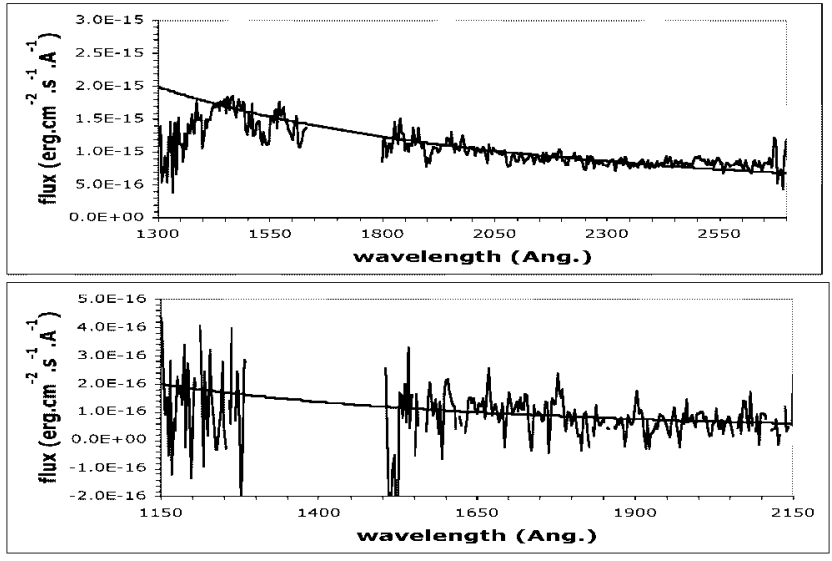

FIG. 1.-One of the best fits of a power law on the spectrum of ELAISC15 J003828-433848 at $z=0.048(\beta=-1.58 \pm 0.04)$ (top) and one of the worst for ELAISC15 J003531-434448 at $z=0.286(\beta=-2.04 \pm 0.27)$ (bottom). Note that because of its redshift, the fit of the latter spectrum was performed on fewer pixels, since we do not use pixels at $\lambda \leq 1200 \AA$.

$z \leq 0.1, \beta$ correlates with FUV - NUV and the trend is very consistent with the GALEX FUV - NUV versus $\beta_{\mathrm{GLX}}$ relationship found by Kong et al. (2004) within an absolute uncertainty of about $0.10-0.15$ mag. To further check this, we have integrated the Kinney et al. (1993) templates into GALEX filters (except the bulge and elliptical templates). The location and standard deviation of these points are consistent with our data for $z<0.1$. For this reason, the wavelength range used for the fit is restricted to rest-frame wavelengths $\lambda>1200 \AA$. We suggest that any estimation of $\beta$ from FUV - NUV should include a $K$-correction above about $z \sim 0.1$ as performed in Schiminovich et al. (2005) and Arnouts et al. (2005).

\section{DUST ATTENUATION AND STAR FORMATION RATES}

By construction, all our objects are detected in UV and in MIR. From the $15 \mu \mathrm{m}$ fluxes, we evaluated the IR bolometric luminosity $L_{\mathrm{IR}}=L(8-1000 \mu \mathrm{m})$ (Chary \& Elbaz 2001) and we estimated the $L_{\mathrm{IR}} / L_{\mathrm{FUv}}$ ratio. The $1 \sigma$ uncertainty for the $15 \mu \mathrm{m}$ flux to IR bolometric luminosity relation is about 0.2 in $\log L_{\mathrm{IR}}$. However, by accounting for measurement errors, it could rise to $0.5-0.7$ in $\log L_{\mathrm{IR}}$.

GALEX spectroscopic observations present the first opportunity of comparing actual spectroscopic $\beta$-values to the $L_{\mathrm{IR}} / L_{\mathrm{FUV}}$ ratio for LIRGs. This is important, because estimates obtained from colors (see, for instance, Meurer et al. 1999 for a discussion of spectroscopic and photometric $\beta$ 's) could lead to larger uncertainties in the evaluation of $\beta$, since the whole spectral energy distribution is binned to two points (with their associated uncertainties). Therefore, we lose the information about lines, the shape of the attenuation curve, and the star formation history necessary to get the best $\beta$. Moreover, we do not need to correct for band-shifting and $K$-corrections (see $\S 3$ ).

The location of our galaxy sample in the $L_{\mathrm{IR}} / L_{\mathrm{FUV}}$ versus $\beta$ diagram, plotted in Figure 3, shows that a simple law cannot represent the whole sample of galaxies. Indeed, if some of them follow the Kong et al. (2004) IR/FUV versus $\beta$ law for starbursts, most of them fall above the relation and suggest a higher extinction that would be evaluated from $\beta$. ULIRGs from Goldader et al. (2002) are also overplotted in this diagram. All but one lie in the top-right zone of the diagram (greater UV attenuations and flatter slopes); these objects are evidently much more extreme than ours, but they seem to have a similar be-

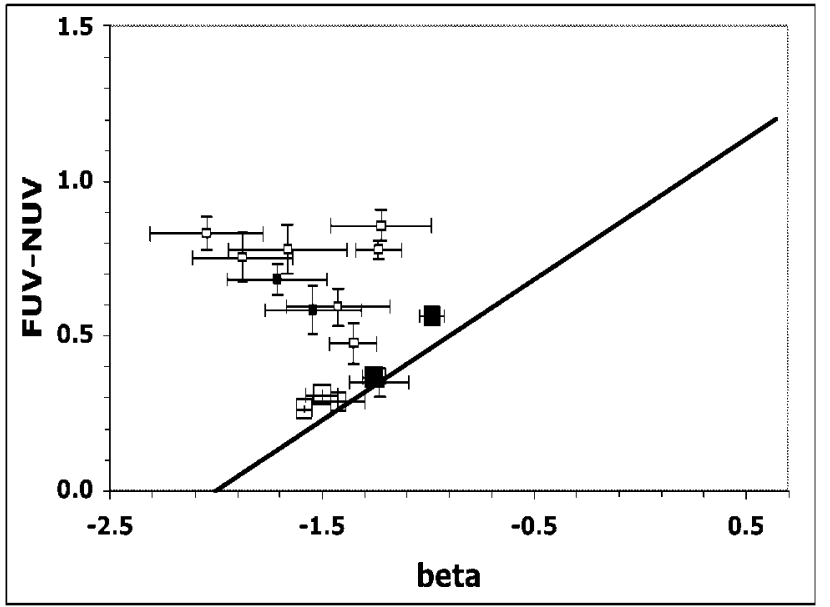

FIG. 2.-GALEX FUV - NUV color plotted against the UV slope $\beta$ estimated from GALEX spectra. Our sample is represented as follows: Open squares are normal galaxies, and filled squares are Seyfert 2 galaxies. We use larger squares for galaxies with $z<0.1$. There is no clear relationship between $\beta$ and FUV - NUV for the whole galaxy sample. However, there is a tight relationship if we use only galaxies at $z<0.1$. Within the GALEX maximal $0.1-0.15$ uncertainty, all the lowest redshift galaxies closely follow the law (solid line) given by Kong et al. (2004) and the Kinney et al. (1993) templates (crosses) integrated into GALEX bandpasses.

havior. It is also interesting to notice that most of the Seyfert 2 galaxies have the highest $L_{\mathrm{IR}} / L_{\mathrm{FUV}}$, which might mean that there is an IR contribution from a dust torus around the QSO (Rowan-Robinson et al. 2004). By using the relation given by Buat et al. (2005), we can estimate the FUV dust attenuation listed in Table 1. The median value for our sample is $A_{\mathrm{FUV}}=2.7 \pm 0.8$, in agreement with the Buat et al. (2005) median value of $A_{\mathrm{FUv}}=2.9_{-1.1}^{+1.3}$ for their far-IR-selected sample and their median value of $A_{\mathrm{FUV}}=1.1_{-0.4}^{+0.5}$ for their near-UV (NUV)-selected sample.

From the spectrum of the nearest galaxy in the sample, ELAISC15 J003828-433848 at $z=0.048$, we can see a trough in the spectrum, which is consistent with being produced by a

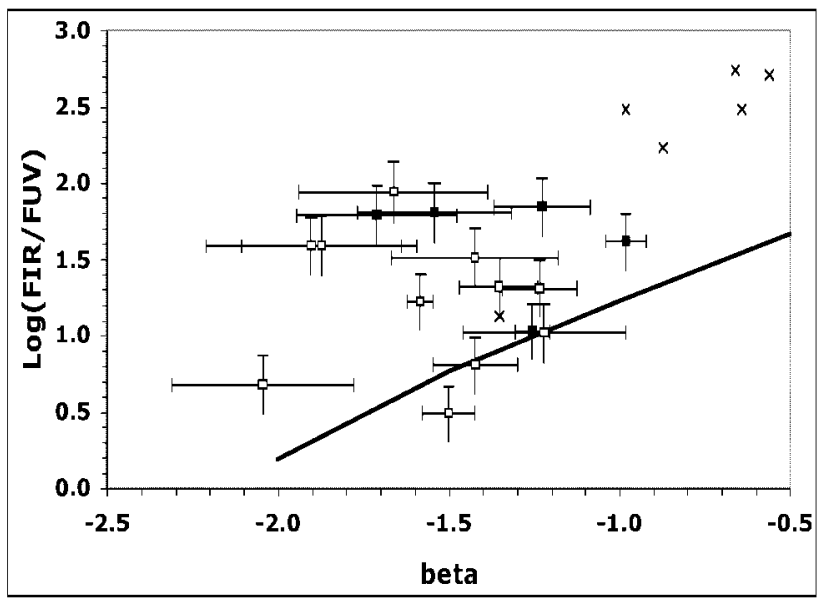

FIG. 3.- $\log$ of the $L_{\mathrm{IR}} / L_{\mathrm{FUV}}$ ratio plotted against the UV slope $\beta$. Our sample is represented with the same symbols as in Fig. 2, and Goldader et al. (2002) objects are added as crosses. One of them falls within our sample, and the other ones are redder and more extinguished. Most of the objects do not follow the law deduced from the UV observation of starbursts (e.g., Kong et al. 2004 overplotted here): the sample extends upward. 
bump in the attenuation law at the same location as the one present in the Milky Way attenuation curve near $220 \mathrm{~nm}$ (Fig. 4). Within the limit of this early calibration of GALEX spectroscopy, the fit to the continuum gives $\beta=-1.58 \pm$ 0.04. Assuming the presence of the $220 \mathrm{~nm}$ bump in this LIRG, we can estimate a new slope by fitting a power law without accounting for wavelengths in the range $200-250 \mathrm{~nm}$, i.e., where the effect of the bump is maximal (e.g., Witt \& Gordon 2000). The new value of the slope is $\beta=-1.26$, i.e., at $8 \sigma$ from the previous value. This galaxy falls above the Kong et al. (2004) law (see Fig. 3), but if we use the new value of $\beta$, the galaxy shifts to the right (shallower $\beta$ ) and gets closer to the curve. Of course, it is difficult to draw conclusions from this single instance, but Motta et al. (2002) suggest the presence of the same feature in the spectrum of a galaxy at $z \sim 0.83$. Moreover, even though the sample is built from normal galaxies and not LIRGs, it is worth noticing that Rowan-Robinson (2003) found that a normal Galactic extinction law seems consistent with their analysis of their data. Finally, the only high-S/N large sample of UV spectra is the Kinney et al. (1993) atlas, which contains some highly reddened starbursts that do not show the $220 \mathrm{~nm}$ feature. Addressing this point would therefore be an important goal for the interpretation of rest-frame UV observations and to get better estimates of the dust attenuation before computing SFRs: we need to analyze a sample of high-S/N GALEX spectra containing several galaxy types.

We list, in Table 1, the SFRs estimated from the Kennicutt (1998) relations. After correction for the dust attenuation in the FUV estimated from the IR/FUV ratio (Buat et al. 2005), both SFRs correlate with a slope of 1.0 and a standard deviation of 6.6 $M_{\odot} \mathrm{yr}^{-1}$ for non-Seyfert galaxies, i.e., for those where the IR flux is only coming from recycled star photons and not from a dust torus that would surround the QSO.

\section{CONCLUSION}

This Letter presents the first UV spectra of LIRGs. The analysis of GALEX spectra with complementary $15 \mu \mathrm{m}$ data from ELAIS allowed us to define a sample of galaxies in the redshift range $0.02 \leq z \leq 0.35$ with multiwavelength data. We show that the raw GALEX UV color FUV - NUV cannot be used to estimate the UV slope $\beta$ for galaxies at redshifts above $z \sim 0.1$ because the GALEX FUV band contains rest-frame flux below $\lambda \sim 1200 \AA$, where spectra flatten. From this multi- $\lambda$ study, we compare two dust attenuation tracers: the UV slope $\beta$ and the

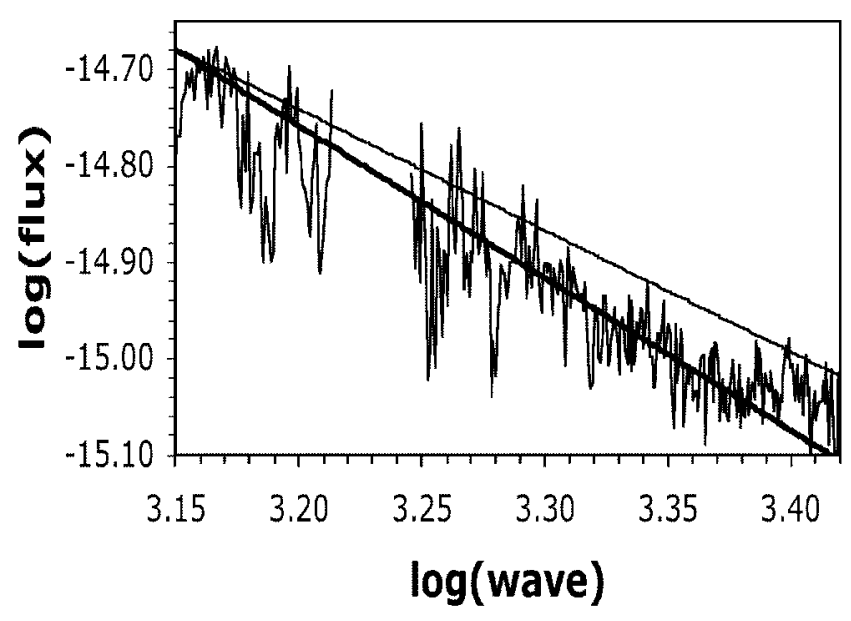

FIG. 4.-Highest quality spectrum is shown with its fits $\left(f_{\lambda} \propto \lambda^{\beta}\right)$. This object seems to exhibit a trough consistent with being due to a bump around $220 \mathrm{~nm}$ ( $\log \lambda=3.34$ ), similar to the Milky Way extinction law. The regular fit (thick line) gives $\beta=-1.58 \pm 0.04$. If we estimate $\beta$ without the trough by excluding pixels in the range $1900-2500 \AA$, i.e., $3.28 \leq \log \lambda \leq 3.39$ (thin line), we obtain $\beta=-1.26$ at $8 \sigma$ from the previous value. The flux is given in units of ergs $\mathrm{s}^{-1} \mathrm{~cm}^{-2} \AA^{-1}$, and $\log \lambda$ is in units of angstroms.

IR/UV ratio. We show that for IR-bright objects with an average dust attenuation in FUV, $A_{\mathrm{FUV}}=2.7 \pm 0.8$, the former does not seem to be valid. However, the best $\mathrm{S} / \mathrm{N}$ galaxy at $z=0.048$ could show a hint of the presence of a bump approximately at the same location as the $2175 \AA$ feature in the Milky Way attenuation curve. If we try to correct for the effect of this bump by not using pixels in the range $2000-2500 \AA$, the galaxy gets closer to the location of the starburst relationship in the IR/FUV versus $\beta$ diagram. The $\mathrm{S} / \mathrm{N}$ and/or available wavelength range prevent us from carrying out the same analysis on the rest of the sample, and it is clear that we need to observe other high$\mathrm{S} / \mathrm{N}$, low-redshift galaxies with GALEX.

GALEX is a NASA Small Explorer, launched in 2003 April. We gratefully acknowledge NASA's support for construction, operation, and science analysis for the GALEX mission, developed in cooperation with the Centre National d'Etudes Spatiales of France and the Korean Ministry of Science and Technology. We also thank the French Programme National Galaxies and the Programme National Cosmologie for their financial support.

\section{REFERENCES}

Arnouts, S., et al. 2005, ApJ, 619, L43

Buat, V., Burgarella, D., Deharveng, J.-M., \& Kunth, D. 2002, A\&A, 393, 33 Buat, V., et al. 2005, ApJ, 619, L51

Bunker, A. J., Stanway, E. R., Ellis, R. S., \& McMahon, R. G. 2004, MNRAS, in press (astro-ph/0403223)

Calzetti, D., Kinney, A. L., \& Storchi-Bergmann, T. 1994, ApJ, 429, 582

Chary, R., \& Elbaz, D. 2001, ApJ, 556, 562

Goldader, J. D., Meurer, G., Heckman, T. M., Seibert, M., Sanders, D. B., Calzetti, D., \& Steidel, C. C. 2002, ApJ, 568, 651

Kennicutt, R. C., Jr. 1998, ARA\&A, 36, 189

Kinney, A. L., Bohlin, R. C., Calzetti, D., Panagia, N., \& Wyse, R. F. G. 1993, ApJS, 86, 5

Kong, X., Charlot, S., Brinchmann, J., \& Fall, S. M. 2004, MNRAS, 349, 769

La Franca, F., et al. 2004, AJ, 127, 3075
Leitherer, C., et al. 1999, ApJS, 123, 3

Lowenthal, J. D., et al. 1997, ApJ, 481, 673

Martin, D. C., et al. 2005, ApJ, 619, L1

Meurer, G. R., Heckman, T. M., \& Calzetti, D. 1999, ApJ, 521, 64

Motta, V., et al. 2002, ApJ, 574, 719

Pello, R., Schaerer, D., Richard, J., Le Borgne, J. F., \& Kneib, J.-P. 2004, A\&A, 416, L35

Rowan-Robinson, M. 2003, MNRAS, 344, 13

Rowan-Robinson, M., et al. 2004, MNRAS, 351, 1290

Schiminovich, D., et al. 2005, ApJ, 619, L47

Steidel, C. C., Giavalisco, M., Pettini, M., Dickinson, M., \& Adelberger, K. L. 1996, ApJ, 462, L17

Witt, A. N., \& Gordon, K. D. 2000, ApJ, 528, 799 